\title{
UNIFORM ERROR ESTIMATES FOR CERTAIN NARROW LAGRANGE FINITE ELEMENTS
}

\author{
N. AL SHENK
}

\begin{abstract}
Error estimates of Dupont and Scott are used to derive uniform error estimates for Lagrange finite elements in $\Re^{n} \quad(n \geq 2)$ under the following conditions: (1) The elements can be arbitrarily narrow in any coordinate direction such that a sufficient number of interpolation points are grouped on lines parallel to that coordinate axis, and (2) the space of approximating functions $F_{T}$ in each element $T$ must include the space of polynomials of degree $\leq m-1$ for some $m \geq 1+n / 2$. If $n$ is odd, this does not cover elements of lowest degree that are normally considered with the shape regularity requirement that the ratio of their outer and inner diameters be bounded. For example, if $n=3$, the usual requirement with shape regularity is that each $F_{T}$ contain all first-degree polynomials. The result of this paper requires that each $F_{T}$ contain all quadratic polynomials, and consequently does not apply to linear (Courant) elements in tetrahedrons or trilinear (tensor) elements in rectangular boxes. Counterexamples in these two cases are included.
\end{abstract}

\section{INTRODUCTION}

Most discussions of uniform a priori error estimates for finite element approximations in $\Re^{n}$ with $n \geq 2$ use the shape-regularity assumption that the ratios of the outer and inner diameters of the elements are bounded, where the outer diameter of an element is the diameter of the smallest disk or sphere containing it and its inner diameter is the diameter of the largest disk or sphere contained in it. For triangular elements in $\Re^{2}$ this is equivalent to assuming that the angles in the triangles are bounded away from zero. I. Babuška and A. K. Aziz [1] showed that for certain Lagrange elements on triangles in $\Re^{2}$, this condition is unnecessarily stringent. (We use the term Lagrange element for an approximation that is determined by values of the function being approximated at a finite number of points, in contrast with Hermite elements which also depend on the interpolated function's derivatives.) They showed that instead of requiring that none of the angles in the triangles be small, it suffices to require that none of them be large, i.e., that all angles be bounded away from $\pi$. P. Jamet [2] obtained uniform estimates for Lagrange elements under the assumptions that (i) the directions of the sides of the elements are not arbitrarily close to being parallel to any hyperplane and (ii) the space of approximating functions in each element include all polynomials of degree $k$ with $k>n / 2$. The last restriction eliminates linear, bilinear, and trilinear elements in $\Re^{2}$ and $\Re^{3}$.

Received by the editor October 7, 1992 and, in revised form, June 22, 1993.

1991 Mathematics Subject Classification. Primary 65N30. 
Later, E. Barnhill and J. A. Gregory [3, 4] derived uniform estimates for linear elements on triangles with angles bounded away from $\pi$, and M. H. Schultz [5, pp. 19-20] obtained such results for certain Lagrange elements on arbitrarily narrow rectangles. Also, L. Dechevski and E. Quak [6] have derived uniform $L^{p}$ estimates for arbitrarily narrow elements in $R^{n}$ with $n<p$.

In this paper we use error estimates of Dupont and Scott [7] for approximations by averaged Taylor polynomials to generalize these results to other types of Lagrange elements in $\Re^{n}$ for $n \geq 2$. The necessary results from [7] require only Schwarz inequalities and changes of variables in multiple integrals. (See also the expository article [8]). We will show that, in most cases, uniform estimates can be obtained for elements that are arbitrarily narrow in any coordinate direction such that a sufficient number of interpolation points are grouped on lines parallel to that coordinate axis, and for the images of such elements under nonsingular linear transformations such that the ratios of their greatest and least singular values are bounded. The main result (Theorem 1) applies to all types of elements covered by standard results under a shape-regularity requirement, with one important exception. It does not apply to elements of the lowest possible degree in spaces of odd dimension $n$. The results based on shape-regularity require that the space of approximating functions $F_{T}$ in each element $T$ include the space $P_{m-1}$ of polynomials of degree $\leq m-1$ for some $m>n / 2$, while Theorem 1 here requires that $F_{T}$ include $P_{m-1}$ for some $m \geq 1+n / 2$. If $n$ is even, these are equivalent conditions. If $n$ is odd, however, the latter condition is stronger. For $n=3$, the requirement with shape-regularity is that each $F_{T}$ contain all first-degree polynomials and Theorem 1 here requires that each $F_{T}$ contain all quadratic polynomials. Consequently, Theorem 1 does not apply to linear (Courant) elements in tetrahedrons or trilinear (tensor) elements in rectangular boxes, and we include counterexamples to Theorem 1 in these two cases.

\section{The FINITE ELEMENTS}

We assume that the reference element $T_{0}$ is a closed polygon in $\Re^{n}$ with $n=2$, or a closed polyhedron in $\Re^{n}$ with $n \geq 3$, and that the domain $\Omega \subset \Re^{n}$ under consideration is a polygon or polyhedron whose closure is paved by a finite number of images $T$ of $T_{0}$ under affine transformations $y_{T}(x)=A_{T}^{t} x+$ $b_{T}$, where $y_{T}$ and $x$ are column vectors and for each $T, A_{T}$ is an $n \times n$ nonsingular matrix, $A_{T}^{t}$ is its transpose, and $b_{T}$ is a constant vector. Here we view the transformation from the reference element $T_{0}$ to the general element $T$ as the composition of three transformations by writing $A_{T}^{t}$ in the form

$$
A_{T}^{t}=h_{T} S_{T}^{t} D_{T},
$$

where $h_{T}$ is a positive constant, $S_{T}$ is an arbitrary real, nonsingular $n \times n$ matrix, and $D_{T}$ is a real, diagonal $n \times n$ matrix whose diagonal elements $d_{r r}$ satisfy the following condition:

I. There is a subset $R$ of $\{1,2, \ldots, n\}$ such that

$$
0<d_{r r}<1 \text { for } r \in R \text { and } d_{r r}=1 \text { for } r \notin R \text {. }
$$

The factor $h_{T}$ in (1) represents a uniform contraction or expansion in all directions, the matrix $D_{T}$ represents contractions parallel to the $x^{r}$-axes for $r \in R$, and $S_{T}$ can involve contractions parallel to the $x^{r}$-axes for $r \notin R$ or 
other nonsingular linear transformations. We will obtain, for a fixed reference element, estimates involving $h_{T}$ and the singular values of $S_{T}$ that are independent of $D_{T}$ and hence uniform for the images under $h_{T} S_{T}^{t}$ of elements that are arbitrarily narrow in the $x^{r}$-directions for $r \in R$.

We also make the following assumptions. Of these, it is Condition VIII that allows for narrow elements.

II. The reference element $T_{0}$ contains an open convex set $U_{0}$ with the property that $T_{0}$ is star-shaped with respect to all points in $U_{0}$.

III. The finite-dimensional space $F_{0}$ of functions $\phi(x)$ that are used as approximations in the reference element $T_{0}$ consists of functions in $C\left(T_{0}\right)$ whose first-order distribution derivatives in the interior of $T_{0}$ are in $L^{2}\left(T_{0}\right)$ and includes all polynomials of degree $\leq m-1$, where $m$ is an integer $\geq 1+n / 2$.

IV. The interpolation data in $T_{0}$ consist of the values $\left\{u\left(p_{j}\right)\right\}$ of the interpolated function at $N$ points $\left\{p_{j}\right\}$ in $T_{0}$, with $N$ the dimension of $F_{0}$ and such that

$$
B_{0} u=\left[u\left(p_{1}\right), u\left(p_{2}\right), \ldots, u\left(p_{N}\right)\right]: F_{0} \rightarrow \Re^{N}
$$

is an isomorphism. The interpolant of $u \in C^{m}\left(T_{0}\right)$ is the unique function $u_{I}$ in $F_{0}$ such that $B_{0} u_{I}=B_{0} u$.

V. The space $F_{T}$ of approximating functions $\psi(y)$ in a general element $T$ is obtained from $F_{0}$ by the affine mapping $y_{T}(x)$; i.e., $F_{T}=\{\psi(y): \tilde{\psi}(x)=$ $\left.\psi\left(y_{T}(x)\right) \in F_{0}\right\}$.

VI. The interpolation data $B_{T} u$ in the general element $T$ are obtained from the data in $T_{0}$ by the mapping $y_{T}(x)$ :

$$
B_{T} u=B_{0} \tilde{u}
$$

with $\tilde{u}(x)=u\left(y_{T}(x)\right)$ for $u(y) \in C^{m}(T)$. This means that

$$
B_{T} u=\left[u\left(q_{1}\right), u\left(q_{2}\right), \ldots, u\left(q_{N}\right)\right]
$$

with $q_{j}=y_{T}\left(p_{j}\right)$. The interpolant of $u(y) \in C^{m}(T)$ is the unique function $u_{I}(y)$ in $F_{T}$ such that $B_{T} u_{I}=B_{T} u$.

VII. The interpolation data is such that the interpolations of a function $u \in$ $C^{m}(\bar{\Omega})$ in the various elements combine to form a function $u_{I} \in C(\bar{\Omega})$.

VIII. For each interpolating point $p_{j}$ in $T_{0}$ and each $r \in R$, let $\phi_{j r}(x)$ be the function in $F_{0}$ that equals 1 at all interpolating points on the line through $p_{j}$ parallel to the the $x^{r}$-axis and equals 0 at all other interpolating points. Then $\phi_{j r}(x)$ is independent of $x^{r}$.

In the case of $n=2$, Condition VIII is satisfied if $F_{0}$ contains the space $P_{m-1}$ of all polynomials of degree $\leq m-1$ and if, for each $r \in R$, the interpolation points $\left\{p_{j}\right\}$ are grouped on $m$ lines parallel to the $x^{r}$-axis. This is a consequence of the fact that a polynomial of degree $\leq m-1$ in one variable can be chosen to have arbitrary values at $m$ points. For example, Figures 1 and 2 (next page) represent triangular elements in the isosceles right triangle $T_{0}$ with vertices $p_{1}=(0,0), p_{2}=(1,0)$, and $p_{3}=(0,1)$. Both types of elements satisfy Condition VIII with $R=\{1,2\}$. In the case of Figure 1, which represents linear (Courant) elements determined by values of the functions at the three vertices, $m=2$ and the interpolation points are on two lines parallel to each coordinate axis. Here, 1 can be in $R$ because the linear function $1-x^{2}$ that is 1 at $p_{1}$ and $p_{2}$ and is 0 at $p_{3}$ and the linear function $x^{2}$ that is 1 


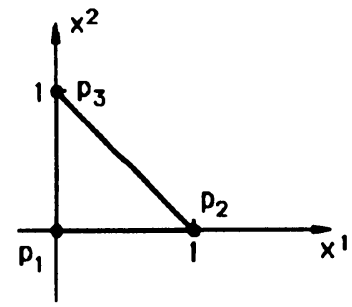

FIGURE 1

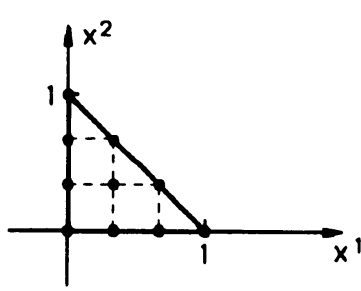

Figure 2

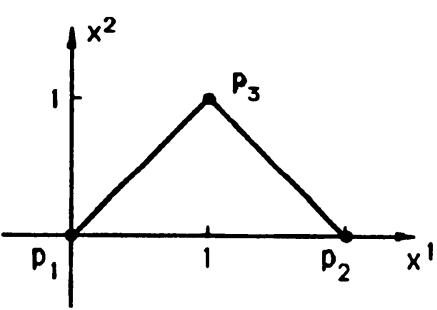

Figure 3

at $p_{3}$ and is 0 at $p_{1}$ and $p_{2}$ are both independent of $x^{1}$. Similarly, 2 can be in $R$ in this case. For cubic elements determined by the ten points in Figure 2, $m=4$ and 1 and 2 can be in $R$ since the ten points are grouped on four horizontal and four vertical lines.

The situation is different for linear elements if $T_{0}$ is the isosceles triangle with vertices $p_{1}=(0,0), p_{2}=(2,0)$, and $p_{3}=(1,1)$ in Figure 3 . In this case, 1 can be in $R$ because $p_{1}, p_{2}$, and $p_{3}$ are on two horizontal lines. The function $x^{2}$ that is 1 at $p_{3}$ and is 0 at $p_{1}$ and $p_{2}$ and the function $1-x^{2}$ that is 1 at $p_{1}$ and $p_{2}$ and is 0 at $p_{3}$ are both independent of $x^{1}$. However, 2 cannot be in $R$ because the linear function $x^{2}$ that is 1 at $p_{3}$ and 0 at $p_{1}$ and $p_{2}$ is not independent of $x^{2}$. The interpolating points are not on two vertical lines. These examples reflect the results of Babuška and Aziz because compressing the triangle of Figure 3 in the $x^{2}$-direction would violate their maximum angle condition, while compressing this triangle in the $x^{1}$-direction or compressing the triangle in Figure 1 and 2 in the $x^{1}$-or $x^{2}$-direction would not.

To deal with the case of general $n \geq 2$ and a fixed $r$, we let

$$
\hat{x}=\left(x^{1}, \ldots, x^{r-1}, x^{r+1}, \ldots, x^{n}\right)
$$

denote the point in $\Re^{n-1}$ obtained by removing $x^{r}$ from $x=\left(x^{1}, x^{2}, \ldots, x^{n}\right)$, and use $\left(\hat{x}, x_{r}\right)$ as alternate notation for $x$. Suppose $\hat{P}_{m-1}$ is the space of polynomials of degree $\leq m-1$ in $\hat{x}$ and $\hat{N}$ is its dimension. Then Condition VIII is satisfied if $F_{0}$ contains $\hat{P}_{m-1}$ and the interpolation points $\left\{x_{j}\right\}$ are grouped on $\hat{N}$ lines $\hat{x}=\hat{c}_{\nu}, \nu=1,2, \ldots, \hat{N}$, such that a polynomial in $\hat{P}_{m-1}$ can be assigned arbitrary values at the points $\left\{\hat{c}_{\nu}\right\} \in \Re^{n-1}$.

\section{NORMS AND SEMINORMS}

For a nonnegative integer $j$ and a function $u$ with continuous derivatives of order $j$ at $x$,

$$
[u(x)]_{x, j}=\left\{\sum_{|\alpha|=j} \frac{j !}{\alpha !}\left|\left(\frac{\partial}{\partial x}\right)^{\alpha} u(x)\right|^{2}\right\}^{1 / 2}
$$

denotes the $\ell^{2}$-norm in $\Re^{n^{j}}$ of the $x$-derivatives of $u$ of order $j$ at $x$, with the mixed partial derivatives obtained by different orders of differentiation counted separately. Here the multi-index $\alpha=\left(\alpha^{1}, \alpha^{2}, \ldots, \alpha^{n}\right)$ is an $n$-tuple of nonnegative integers, $|\alpha|=\alpha^{1}+\alpha^{2}+\cdots+\alpha^{n}, \alpha !=\alpha^{1} ! \alpha^{2 !} \cdots \alpha^{n} !$ and

$$
\left(\frac{\partial}{\partial x}\right)^{\alpha}=\frac{\partial^{|\alpha|}}{\left(\partial x^{1}\right)^{\alpha_{1}} \cdots\left(\partial x^{n}\right)^{\alpha_{n}}} \text {. }
$$


For $j \geq 0$,

$$
|u|_{T, j}=\left\{\int_{T}[u(x)]_{x, j}^{2} d x\right\}^{1 / 2}
$$

is the seminorm (a norm if $j=0$ ) formed by the $L^{2}$-norms of the $j$ th derivatives of $u$ in $T$, and

$$
\|u\|_{T, k}=\left\{\sum_{j=0}^{k}|u|_{T, j}^{2}\right\}^{1 / 2}
$$

is a norm in $L^{2}(T)$ for $k=0$ and in the Sobolev space $H^{1}(T)$ for $k=1$.

\section{The MaIN Result}

Theorem 1. Suppose that the reference data-the reference element $T_{0}$, its subset $U_{0}$, the integer $m$, the space of approximating functions $F_{0}$, and the interpolation points $\left\{p_{j}\right\}$-satisfy hypotheses (II) through (VIII) above.

Let $h_{T}$ be a positive constant, $S_{T}$ a nonsingular $n \times n$ matrix, $D_{T}$ an $n \times n$ diagonal matrix satisfying hypothesis $(\mathrm{I})$, and $b_{T}$ a constant vector in $\Re^{n}$. Let $T$ be the image of $T_{0}$ under the mapping $y_{T}=h_{T} S_{T}^{t} D_{T} x+b_{T}$.

Then there is a constant $C$, that depends only on the reference data in $T_{0}$, such that for all $u \in C^{m}(T)$,

$$
\left|u-u_{I}\right|_{T, 0}^{2}+\left(h_{T} \lambda_{T}\right)^{2}\left|u-u_{I}\right|_{T, 1}^{2} \leq C^{2}\left(h_{T} \Lambda_{T}\right)^{2 m}|u|_{T, m}^{2},
$$

where $u_{I}$ is the interpolant of $u$ in $T$ and $\lambda_{T}$ and $\dot{\Lambda}_{T}$ are the least and greatest singular values of $S_{T}$.

If $\Lambda_{T} / \lambda_{T}$ is bounded for all elements $T$ under consideration, then the factors $\Lambda_{T}$ and $\lambda_{T}$ can be dropped from (8)-with a different constant $C$.

Throughout this discussion, $C$ denotes various constants that depend only on the reference data in $T_{0}$. By the standard error estimates for $T_{0}$, there is a constant $C$ such that

$$
\left|u-u_{I}\right|_{T_{0}, 0}^{2}+\left|u-u_{I}\right|_{T_{0}, 1}^{2} \leq C^{2}|u|_{T_{0}, m}^{2} \text { for } u \in C^{m}\left(T_{0}\right) .
$$

We rewrite this, using only $L^{2}$-norms, as

$$
\left|u-u_{I}\right|_{T_{0}, 0}^{2}+\sum_{j=1}^{n}\left|\frac{\partial}{\partial x^{j}}\left(u-u_{I}\right)\right|_{T_{0}, 0}^{2} \leq C^{2} \sum_{|\alpha|=m} \frac{m !}{\alpha !}\left|\left(\frac{\partial}{\partial x}\right)^{\alpha} u\right|_{T_{0}, 0}^{2} .
$$

Let $T_{1}$ be the image of $T_{0}$ under the mapping $z=D x$, which compresses the $x^{r}$-directions for $r \in R$. Under this change of variables,

$$
\frac{\partial}{\partial x^{j}}=\frac{\partial z^{j}}{\partial x^{j}} \frac{\partial}{\partial z^{j}}=d_{j j} \frac{\partial}{\partial z^{j}}
$$

so that (9) becomes, after cancelling the Jacobian, $\operatorname{det}(D)^{-1}$,

$$
\left|u-u_{I}\right|_{T_{1}, 0}^{2}+\sum_{j=1}^{n}\left|d_{j j} \frac{\partial}{\partial z^{j}}\left(u-u_{I}\right)\right|_{T_{1}, 0}^{2} \leq C^{2} \sum_{|\alpha|=m} \frac{m !}{\alpha !}\left|d^{\alpha}\left(\frac{\partial}{\partial z}\right)^{\alpha} u\right|_{T_{1}, 0}^{2}
$$


with $d^{\alpha}=d_{11}^{\alpha^{1}} \cdots d_{n n}^{\alpha^{n}}$. Since $\left|d_{j j}\right| \leq 1$ for $j=1,2, \ldots, n$, we have $\left|d^{\alpha}\right| \leq 1$ and

$$
\left|u-u_{I}\right|_{T_{1}, 0}^{2}+\sum_{j=1}^{n}\left|d_{j j} \frac{\partial}{\partial z^{j}}\left(u-u_{I}\right)\right|_{T_{1}, 0}^{2} \leq C^{2} \sum_{|\alpha|=m} \frac{m !}{\alpha !}\left|\left(\frac{\partial}{\partial z}\right)^{\alpha} u\right|_{T_{1}, 0}^{2}
$$

or

$$
\left|u-u_{I}\right|_{T_{1}, 0}^{2}+\sum_{j=1}^{n}\left|d_{j j} \frac{\partial}{\partial z^{j}}\left(u-u_{I}\right)\right|_{T_{1}, 0}^{2} \leq C^{2}|u|_{T_{1}, m}^{2}
$$

for $u \in C^{m}\left(T_{1}\right)$.

Estimate (11) by itself will not give Theorem 1 because $\left|d_{r r}\right|$ can be arbitrarily small for $r \in R$. We also need the following result.

Lemma 1. There is a constant $C$, depending only on the reference data in $T_{0}$, such that for $u \in C^{m}\left(T_{0}\right)$ and $r \in R$,

$$
\left|\frac{\partial}{\partial x^{r}}\left(u-u_{I}\right)\right|_{T_{0}, 0}^{2} \leq C^{2}\left|\frac{\partial u}{\partial x^{r}}\right|_{T_{0}, m-1}^{2} .
$$

Suppose that Lemma 1 has been established. We rewrite (12) with $L^{2}$-norms as

$$
\left|\frac{\partial}{\partial x^{r}}\left(u-u_{I}\right)\right|_{T_{0}, 0}^{2} \leq C^{2} \sum_{|\alpha|=m-1} \frac{(m-1) !}{\alpha !}\left|\left(\frac{\partial}{\partial x}\right)^{\alpha} \frac{\partial u}{\partial x^{r}}\right|_{T_{0}, 0}^{2} .
$$

Then the change of variables $z=D x$ yields

$$
\left|d_{r r} \frac{\partial}{\partial z^{r}}\left(u-u_{I}\right)\right|_{T_{1}, 0}^{2} \leq C^{2} \sum_{|\alpha|=m-1} \frac{(m-1) !}{\alpha !}\left|d^{\alpha} d_{r r}\left(\frac{\partial}{\partial z}\right)^{\alpha} \frac{\partial u}{\partial z^{r}}\right|_{T_{1}, 0}^{2}
$$

We cancel $\left|d_{r r}\right|^{2}$ and use the estimate $\left|d^{\alpha}\right| \leq 1$ to obtain for $r \in R$,

$$
\begin{aligned}
\left|\frac{\partial}{\partial z^{r}}\left(u-u_{I}\right)\right|_{T_{1}, 0}^{2} & \leq C^{2} \sum_{|\alpha|=m-1} \frac{(m-1) !}{\alpha !}\left|\left(\frac{\partial}{\partial z}\right)^{\alpha} \frac{\partial u}{\partial z^{r}}\right|_{T_{1}, 0}^{2} \\
& \leq C^{2} \sum_{|\alpha|=m} \frac{m !}{\alpha !}\left|\left(\frac{\partial}{\partial z}\right)^{\alpha} u\right|_{T_{1}, 0}^{2}=C^{2}|u|_{T_{1}, m}^{2} .
\end{aligned}
$$

Deleting the terms in the sum of (11) with $j=r \in R$, for which $d_{r r}<1$, and replacing them by (13) yields

$$
\left|u-u_{I}\right|_{T_{1}, 0}^{2}+\sum_{j=1}^{n}\left|\frac{\partial}{\partial z^{j}}\left(u-u_{I}\right)\right|_{T_{1}, 0}^{2} \leq C^{2}|u|_{T_{1}, m}^{2}
$$

or

$$
\left|u-u_{I}\right|_{T_{1}, 0}^{2}+\left|u-u_{I}\right|_{T_{1}, 1}^{2} \leq C^{2}|u|_{T_{1}, m}^{2} .
$$

Making the change of variables $y=h_{T} S_{T}^{t} z+b_{T}$ yields (8) (see Lemma 3 of [8]). Hence we can establish Theorem 1 by proving Lemma 1. 


\section{Proof of Lemma 1}

For $u \in C^{m}\left(T_{0}\right)$, let $Q^{m} u$ be the averaged Taylor polynomial approximation of $u$ of degree $m-1$ defined in equation (3.1) of [7] with $\Omega=T_{0}$ and $B=U_{0}$. We will estimate $\frac{\partial}{\partial x^{r}}\left(u-u_{I}\right)$ for $r \in R$ by estimating $\frac{\partial}{\partial x^{r}}\left(u-Q^{m} u\right)$ and $\frac{\partial}{\partial x^{r}}\left(Q^{m} u-u_{I}\right)$. Equation (3.3) and the last set of inequalities on p. 38 of [7] with $|\alpha|=0, j=0, u$ replaced by $\frac{\partial u}{\partial x^{r}}$, and $m$ replaced by $m-1$ give

$$
\begin{aligned}
\left|\frac{\partial}{\partial x^{r}}\left(u-Q^{m} u\right)\right|_{T_{0}, 0} & =\left|\frac{\partial u}{\partial x^{r}}-Q^{m-1} \frac{\partial u}{\partial x^{r}}\right|_{T_{0}, 0} \\
& =\left|R^{m-1} \frac{\partial u}{\partial x_{r}}\right|_{T_{0}, 0} \leq C\left|\frac{\partial u}{\partial x^{r}}\right|_{T_{0}, m-1}
\end{aligned}
$$

This is the estimate we need on $\frac{\partial}{\partial x^{r}}\left(u-Q^{m} u\right)$.

Since $Q^{m} u$ is a polynomial of degree $\leq m-1$, it and $u_{I}-Q^{m} u$ are in $F_{0}$ by Condition III. We begin our study of $u_{I}-Q^{m}$ by looking at functions in $F_{0}$.

We suppose that $r \in R$ is fixed. We let $\hat{x}=\hat{c}_{\nu}, \nu=1,2,3, \ldots, \hat{N}$, be the lines parallel to the $x^{r}$-axis containing the interpolating points $\left\{p_{j}\right\}$ in $T_{0}$, and let $\left(\hat{c}_{\nu}, t_{\nu \mu}\right)$ for $\mu=1,2, \ldots, M_{\nu}$ be the interpolating points on $\hat{x}=\hat{c}_{\nu}$. Label the points so that $t_{\nu 1}<t_{\nu \mu}$ for $\mu>1$. Then $\left(\hat{c}_{\nu}, t_{\nu \mu}\right)$ for $\mu=1,2, \ldots, M_{\nu}$ and $\nu=1,2, \ldots, \hat{N}$ are all the interpolating points. The next lemma uses Condition VIII on the geometry of those points.

Lemma 2. There is a constant $C$, depending only on the reference data, such that for $\phi \in F_{0}$ and $r \in R$,

$$
\int_{T_{0}}\left|\frac{\partial \phi}{\partial x^{r}}(x)\right|^{2} d x \leq C^{2} \sum_{\nu=1}^{\hat{N}} \sum_{\mu=2}^{M_{\nu}}\left|\phi\left(\hat{c}_{\nu}, t_{\nu \mu}\right)-\phi\left(\hat{c}_{\nu}, t_{\nu 1}\right)\right|^{2}
$$

where the inner sum is taken to be zero if $M_{\nu}=1$.

Proof. For each interpolation point $\left(\hat{c}_{\nu}, t_{\nu \mu}\right)$, let $\chi_{\nu \mu}(x)$ be the function in $F_{0}$ that equals 1 at $\left(\hat{c}_{\nu}, t_{\nu \mu}\right)$ and equals zero at the other interpolation points. Then

$$
\phi(x)=\sum_{\nu=1}^{\hat{N}} \sum_{\mu=1}^{M_{\nu}} \phi\left(\hat{c}_{\nu}, t_{\nu \mu}\right) \chi_{\nu \mu}(x) .
$$

By Hypothesis VIII, the functions $\sum_{\mu=1}^{M_{\nu}} \chi_{\nu \mu}(x)$ for $\nu=1,2, \ldots, \hat{N}$ are independent of $x^{r}$. Consequently, their $x^{r}$-derivatives are zero and

$$
\frac{\partial}{\partial x^{r}} \chi_{\nu 1}(x)=\left\{\begin{array}{lll}
0 & \text { if } & M_{\nu}=1 \\
-\sum_{\mu=2}^{M_{\nu}} \frac{\partial}{\partial x^{r}} \chi_{\nu \mu}(x) & \text { if } & M_{\nu}>1
\end{array}\right\}=-\sum_{\mu=2}^{M_{\nu}} \frac{\partial}{\partial x^{r}} \chi_{\nu \mu}(x),
$$


where the sum is taken to be zero if $M_{\nu}=1$. Using this with (16) gives

$$
\begin{aligned}
\frac{\partial \phi}{\partial x^{r}}(x) & =\sum_{\nu=1}^{\hat{N}} \sum_{\mu=1}^{M_{\nu}} \phi\left(\hat{c}_{\nu}, t_{\nu \mu}\right) \frac{\partial}{\partial x^{r}} \chi_{\nu \mu}(x) \\
& =\sum_{\nu=1}^{\hat{N}}\left\{\phi\left(\hat{c}_{\nu}, t_{\nu 1}\right) \frac{\partial}{\partial x^{r}} \chi_{\nu 1}(x)+\sum_{\mu=2}^{M_{\nu}} \phi\left(\hat{c}_{\nu}, t_{\nu \mu}\right) \frac{\partial}{\partial x^{r}} \chi_{\nu \mu}(x)\right\} \\
& =\sum_{\nu=1}^{\hat{N}}\left\{-\phi\left(\hat{c}_{\nu}, t_{\nu 1}\right) \sum_{\mu=2}^{M_{\nu}} \frac{\partial}{\partial x^{r}} \chi_{\nu \mu}(x)+\sum_{\mu=2}^{M_{\nu}} \phi\left(\hat{c}_{\nu}, t_{\nu \mu}\right) \frac{\partial}{\partial x^{r}} \chi_{\nu \mu}(x)\right\} \\
& =\sum_{\nu=1}^{N} \sum_{\mu=2}^{M_{\nu}}\left[\phi\left(\hat{c}_{\nu}, t_{\nu \mu}\right)-\phi\left(\hat{c}_{\nu}, t_{\nu 1}\right)\right] \frac{\partial}{\partial x^{r}} \chi_{\nu \mu}(x),
\end{aligned}
$$

and then with a Schwarz inequality,

$$
\left|\frac{\partial \phi}{\partial x^{r}}(x)\right|^{2} \leq \sum_{\nu=1}^{\hat{N}} \sum_{\mu=2}^{M_{\nu}}\left|\phi\left(\hat{c}_{\nu}, t_{\nu \mu}\right)-\phi\left(\hat{c}_{\nu}, t_{\nu 1}\right)\right|^{2} \sum_{\nu=1}^{\hat{N}} \sum_{\mu=2}^{M_{\nu}}\left|\frac{\partial}{\partial x^{r}} \chi_{\nu \mu}(x)\right|^{2} .
$$

This yields (15) and establishes Lemma 2 since, by Condition III, $\frac{\partial}{\partial x^{r}} \chi_{\nu \mu}(x)$ are fixed functions in $L^{2}\left(T_{0}\right)$.

To complete the proof of Lemma 1, we use (15) with $\phi=u_{I}-Q^{m} u$. Since $u=u_{I}$ at the interpolation points $\left(\tilde{c}_{\nu}, t_{\nu \mu}\right)$ and $\left(\hat{c}_{\nu}, t_{\nu 1}\right)$ and $\left(\hat{c}_{\nu}, t_{\nu \mu}\right)$ are on the same line parallel to the $x^{r}$-axis, we obtain

$$
\begin{aligned}
\int_{T_{0}} \mid & \left.\frac{\partial}{\partial x^{r}}\left(u_{I}-Q^{m} u\right)(x)\right|^{2} d x \\
& \leq C^{2} \sum_{\nu=1}^{\hat{N}} \sum_{\mu=2}^{M_{\nu}}\left|\left(u_{I}-Q^{m} u\right)\left(\hat{c}_{\nu}, t_{\nu \mu}\right)-\left(u_{I}-Q^{m} u\right)\left(\hat{c}_{\nu}, t_{\nu 1}\right)\right|^{2} \\
& =C^{2} \sum_{\nu=1}^{\hat{N}} \sum_{\mu=2}^{M_{\nu}}\left|\left(u-Q^{m} u\right)\left(\hat{c}_{\nu}, t_{\nu \mu}\right)-\left(u-Q^{m} u\right)\left(\hat{c}_{\nu}, t_{\nu 1}\right)\right|^{2} \\
& =C^{2} \sum_{\nu=1}^{N} \sum_{\mu=2}^{M_{\nu}}\left|\int_{t_{\nu 1}}^{t_{\nu \mu}} \frac{\partial}{\partial x^{r}}\left(u-Q^{m} u\right)\left(\hat{c}_{\nu}, t\right) d t\right|^{2} . \\
& \leq C^{2} \sum_{\nu=1}^{N} \sum_{\mu=2}^{M_{\nu}}\left|\int_{t_{\nu 1}}^{t_{\nu \mu}}\right| \frac{\partial}{\partial x^{r}}\left(u-Q^{m} u\right)\left(\hat{c}_{\nu}, t\right)|d t|^{2} .
\end{aligned}
$$

We use the following lemma to estimate the integrals on the right of (17).

Lemma 3. There is a constant $C$ such that for $\nu=1,2, \ldots, \hat{N}, \mu=1,2, \ldots$, $M_{\nu}$, and $u \in C^{m}\left(T_{0}\right)$,

$$
\left|\int_{t_{\nu 1}}^{t_{\nu \mu}}\right| \frac{\partial}{\partial x^{r}}\left(u-Q^{m} u\right)\left(\hat{c}_{\nu}, t\right)|d t| \leq C\left|\frac{\partial u}{\partial x^{r}}\right|_{T_{0}, m-1}
$$


Proof. We are assuming that $m \geq 1+n / 2$. For $m>1+n / 2$ the first inequality in the proof of Theorem 4.2 in [7] with $u$ replaced by $\frac{\partial u}{\partial x^{r}}, \alpha=0$ and $m$ replaced by $m-1$ gives for $\left(\hat{c}_{\nu}, t\right) \in T_{0}$,

$$
\left|\frac{\partial}{\partial x^{r}}\left(u-Q^{m} u\right)\left(\hat{c}_{\nu}, t\right)\right|=\left|\frac{\partial u}{\partial x^{r}}(x)-Q^{m-1}\left(\frac{\partial u}{\partial x^{r}}\right)\left(\hat{c}_{\nu}, t\right)\right| \leq C\left|\frac{\partial u}{\partial x^{r}}\right|_{T_{0}, m-1} .
$$

Integrating this inequality yields (18).

We are left with the case of $m=1+n / 2$ (and even $n$ ), for which $m-1-n=$ $-n / 2$. The first inequality at the bottom of p. 40 of [7] yields for $x \in T_{0}$, with $D^{\alpha}=\partial / \partial x^{r},|\alpha|=1$, and $\zeta$ in place of $y$,

$$
\begin{aligned}
\mid \frac{\partial}{\partial x^{r}} & \left(u-Q^{m} u\right)(x) \mid \\
& \leq C \int_{T_{0}}|x-\zeta|^{m-1-n} \sum_{|\beta|=m-1} \frac{1}{\beta !}\left|\left(\frac{\partial}{\partial x}\right)^{\beta} \frac{\partial u}{\partial x^{r}}(\zeta)\right| d \zeta \\
& \leq C \int_{T_{0}}|x-\zeta|^{-n / 2}\left[\frac{\partial u}{\partial x^{r}}(\zeta)\right]_{\zeta, m-1} d \zeta .
\end{aligned}
$$

For $x=\left(\hat{c}_{\nu}, t\right)$ and $\zeta=\left(\hat{\zeta}, \zeta^{r}\right)$, set $a=\left|\hat{c}_{\nu}-\hat{\zeta}\right|, \sigma=\left(t-\zeta^{r}\right) / a$, and $d t=a d \sigma$. We have assumed that $t_{\nu 1}<t_{\nu \mu}$ for $\mu>1$. Hence, for $\hat{\zeta} \neq \hat{c}_{\nu}$,

$$
\begin{aligned}
\int_{t_{\nu 1}}^{t_{\nu \mu}} \mid x & -\left.\zeta\right|^{-n / 2} d t=\int_{t_{\nu 1}}^{t_{\nu \mu}}\left[\left|\hat{c}_{\nu}-\hat{\zeta}\right|^{2}+\left(t-\zeta^{r}\right)^{2}\right]^{-n / 4} d t \\
& =\int_{t_{\nu 1}}^{t_{\nu \mu}}\left[a^{2}+\left(t-\zeta^{r}\right)^{2}\right]^{-n / 4} d t \\
& =a^{-n / 2} \int_{t_{\nu 1}}^{t_{\nu \mu}}\left[1+\left(\frac{t-\zeta^{r}}{a}\right)^{2}\right]^{-n / 4} d t \\
& =a^{1-n / 2} \int_{\left(t_{\nu 1}-\zeta^{r}\right) / a}^{\left(t_{\nu \mu}-\zeta^{r}\right) / a}\left[1+\sigma^{2}\right]^{-n / 4} d \sigma \\
& \leq a^{1-n / 2} \int_{-d_{1} / a}^{d_{1} / a}\left[1+\sigma^{2}\right]^{-n / 4} d \sigma=K(a)=K\left(\left|\hat{c}_{\nu}-\hat{\zeta}\right|\right),
\end{aligned}
$$

where $d_{1}$ is the outer diameter of $T_{0}$ and $K(a)$ is defined by the last equations. For $n=2$ and $0<a \leq d_{1}$,

$$
\begin{aligned}
K(a) & =\int_{-d_{1} / a}^{d_{1} / a}\left[1+\sigma^{2}\right]^{-1 / 2} d \sigma=2\left[\ln \left(d_{1}+\sqrt{a^{2}+d_{1}^{2}}\right)-\ln a\right] \\
& \leq C(1+|\ln a|),
\end{aligned}
$$

while for $n \geq 4$,

$$
K(a) \leq a^{1-n / 2} \int_{-\infty}^{\infty}\left[1+\sigma^{2}\right]^{-n / 4} d \sigma \leq C a^{1-n / 2}
$$

Hence for $n=2$,

$$
\left.\int_{T_{0}} K\left(\left|\hat{c}_{\nu}-\hat{\zeta}\right|\right)^{2} d \zeta \leq C \int_{T_{0}}\left(1+|\ln | \hat{c}_{\nu}-\hat{\zeta}\right) \mid\right)^{2} d \zeta \leq C
$$


and for even $n \geq 4$,

$$
\int_{T_{0}} K\left(\left|\hat{c}_{\nu}-\hat{\zeta}\right|\right)^{2} d \zeta \leq C \int_{T_{0}}\left|\hat{c}_{\nu}-\hat{\zeta}\right|^{2-n} d \zeta \leq C .
$$

The integrals on the right of (21a) and (21b) are bounded because their integrands have bounded integrals over the intersections of $T_{0}$ with the $(n-1)$ dimensional planes $x^{r}=c$. Hence, (19) with $x=\left(\hat{c}_{\nu}, t\right),(20)$, and $(21 \mathrm{a}, \mathrm{b})$ yield

$$
\begin{aligned}
\left|\int_{t_{\nu 1}}^{t_{\nu \mu}}\right| \frac{\partial}{\partial x^{r}} & \left(u-Q^{m} u\right)\left(\hat{c}_{\nu}, t\right)|d t|^{2} \\
& \leq C^{2}\left|\int_{T_{0}}\left[\frac{\partial u}{\partial x^{r}}(\zeta)\right]_{\zeta, m-1} \int_{t_{\nu 1}}^{t_{\nu \mu}}\right| x-\left.\left.\zeta\right|^{-n / 2} d t d \zeta\right|^{2} \\
& \leq C^{2}\left|\int_{T_{0}} K\left(\left|\hat{c}_{\nu}-\hat{\zeta}\right|\right)\left[\frac{\partial u}{\partial x^{r}}(\zeta)\right]_{\zeta, m-1} d \zeta\right|^{2} \\
& \leq C^{2} \int_{T_{0}} K\left(\left|\hat{c}_{\nu}-\hat{\zeta}\right|\right)^{2} d \zeta \int_{T_{0}}\left[\frac{\partial u}{\partial x^{r}}(\zeta)\right]_{\zeta, m-1}^{2} d \zeta \leq C^{2}\left|\frac{\partial u}{\partial x^{r}}\right|_{T_{0}, m-1}^{2},
\end{aligned}
$$

and this gives Lemma 3.

Estimates (17) and (18) imply

$$
\begin{aligned}
\left|\frac{\partial}{\partial x^{r}}\left(u_{I}-Q^{m} u\right)\right|_{T_{0}, 0} & =\left\{\int_{T_{0}}\left|\frac{\partial}{\partial x^{r}}\left(u_{I}-Q^{m} u\right)(x)\right|^{2} d x\right\}^{1 / 2} \\
& \leq C\left|\frac{\partial u}{\partial x^{r}}\right|_{T_{0}, m-1}
\end{aligned}
$$

Finally, (14) and (22) with the triangle inequality give Lemma 1 to complete the proof of Theorem 1.

\section{Counterexamples}

We close by using a standard counterexample to the false Sobolev-type inequality $|\psi(0)| \leq C\|\psi\|_{\Re^{2}, 1}$ for functions in $\Re^{2}$ to construct counterexamples to Theorem 1 (i) for $T_{0}$ the tetrahedron in $\Re^{3}$ with vertices $p_{1}=(0,0,0)$, $p_{2}=(1,0,0), p_{3}=(0,1,0), p_{4}=(0,0,1)$ and $F_{0}$ the space of linear functions $\phi=c_{0}+c_{1} x^{1}+c_{2} x^{2}+c_{3} x^{3}$; and (ii) for $T_{0}$ the cube $0 \leq x^{1}, x^{2}, x^{3} \leq 1$ with $F_{0}$ the space of trilinear functions spanned by the eight functions $x^{1} x^{2} x^{3}$, $\left(1-x^{1}\right) x^{2} x^{3}, x^{1}\left(1-x^{2}\right) x^{3},\left(1-x^{1}\right)\left(1-x^{2}\right) x^{3}, x^{1} x^{2}\left(1-x^{3}\right),\left(1-x^{1}\right) x^{2}\left(1-x^{3}\right)$, $x^{1}\left(1-x^{2}\right)\left(1-x^{2}\right)$, and $\left(1-x^{1}\right)\left(1-x^{2}\right)\left(1-x^{3}\right)$.

We set $R=\{3\}$ and write

$$
D_{T}=\left[\begin{array}{lll}
1 & 0 & 0 \\
0 & 1 & 0 \\
0 & 0 & \mu
\end{array}\right]
$$

with $0<\mu<1$, so that $d_{11}=1, d_{22}=1$, and $d_{33}=\mu$. We take $h_{T}=1$ and $b_{T}=0$ and let $S_{T}$ be the identity matrix.

In the case of a tetrahedron $T_{0}$, its image under the mapping $y_{T}=D_{T} x$ is the tetrahedron $T^{\mu}$ with vertices $q_{1}=(0,0,0), q_{2}=(1,0,0), q_{3}=(0,1,0)$ and $q_{4}=(0,0, \mu)$. In the case of a cube, the image of $T_{0}$ is the box $Q^{\mu}$ : 
$0 \leq x^{1} \leq 1,0 \leq x^{2} \leq 1,0 \leq x^{3} \leq \mu$. In both cases all of the hypotheses of Theorem 1 are valid except the requirement in Condition III that $F_{0}$ include all quadratic polynomials.

We consider the case of the tetrahedron first. For $u \in C^{2}\left(T^{\mu}\right), u_{I}$ is the linear function that has the same values as $u$ at $q_{1}, q_{2}, q_{3}, q_{4}$. If Theorem 1 were valid in this case, there would be a constant $C$, independent of $\mu$ with $0<\mu<1$, such that

$$
\left\|u-u_{I}\right\|_{T^{\mu}, 1} \leq C|u|_{T^{\mu}, 2}
$$

for all $u \in C^{2}\left(T^{\mu}\right)$. We will prove there is no such constant by finding for each $Y>0$ a $\mu$ with $0<\mu<1$ and a function $u \in C^{\infty}\left(\Re^{3}\right)$ such that

$$
\left\|u-u_{I}\right\|_{T^{\mu}, 1}>Y|u|_{T^{\mu}, 2} \text {. }
$$

Let $\xi=\left(\xi^{1}, \xi^{2}\right)$ be the Fourier transform variable in $\Re^{2}$ and write $\rho=|\xi|$. Let $\psi_{R}\left(x^{1}, x^{2}\right)$ be the function in $C^{\infty}\left(\Re^{2}\right)$ whose Fourier transform is the radially symmetric function

$$
\mathbf{F}\left(\psi_{R}\right)(\xi)=\left\{\begin{array}{cl}
\frac{1}{\rho^{2} \ln \rho} & \text { for } e<\rho<R, \\
0 & \text { otherwise, }
\end{array}\right.
$$

where $e$ is the base of the natural logarithm and $R$ is a constant $>e$ to be chosen later. Then

$$
\psi_{R}\left(x^{1}, x^{2}\right)=\frac{1}{2 \pi} \int_{\Re^{2}} e^{i\left(x^{1} \xi^{1}+x^{2} \xi^{2}\right)} \mathbf{F}\left(\psi_{R}\right)(\xi) d \xi,
$$

so that

$$
\psi_{R}(0,0)=\int_{e}^{R} \frac{1}{\rho \ln \rho} d \rho=\ln (\ln R)
$$

Also,

$$
\begin{aligned}
\left\|\psi_{R}\right\|_{\Re^{2}, 1}^{2} & =\int_{\Re^{2}}\left|\mathbf{F}\left(\psi_{R}\right)(\xi)\right|^{2}\left(1+|\xi|^{2}\right) d \xi=2 \pi \int_{e}^{R} \frac{1+\rho^{2}}{\rho^{4}(\ln \rho)^{2}} \rho d \rho \\
& <4 \pi \int_{e}^{R} \frac{1}{\rho(\ln \rho)^{2}} d \rho=4 \pi\left[1-\frac{1}{\ln R}\right]<4 \pi
\end{aligned}
$$

and

$$
\begin{aligned}
\left|\psi_{R}\right|_{\Re^{2}, 2}^{2} & =\int_{\Re^{2}}\left|\mathbf{F}\left(\psi_{R}\right)(\xi)\right|^{2}\left(\left|\xi^{1}\right|^{2}+\left|\xi^{2}\right|^{2}\right)^{2} d \xi=2 \pi \int_{e}^{R} \frac{\rho^{4}}{\rho^{4}(\ln \rho)^{2}} \rho d \rho \\
& <2 \pi \int_{e}^{R} \rho d \rho<\pi R^{2} .
\end{aligned}
$$

Define

$$
u\left(x^{1}, x^{2}, x^{3}\right)=\frac{x^{3}}{\sqrt{\mu}} \psi_{R}\left(x^{1}, x^{2}\right) .
$$

Then $u\left(x^{1}, x^{2}, 0\right)=0$ for all $\left(x^{1}, x^{2}\right)$ and $u(0,0, \mu)=\sqrt{\mu} \psi_{R}(0,0)$, so the linear interpolation of $u$ in the tetrahedron $T^{\mu}$ is

$$
u_{I}\left(x^{1}, x^{2}, x^{3}\right)=\frac{x^{3}}{\sqrt{\mu}} \psi_{R}(0,0) .
$$


Since the $x^{1}$ - and $x^{2}$-derivatives of $u_{I}$ are zero and its $x^{3}$-derivative is $\psi_{R}(0,0) / \sqrt{\mu}$, we have

$$
\begin{aligned}
\left\|u_{I}\right\|_{T^{\mu}, 1}^{2} & =\frac{1}{\mu} \int_{T^{\mu}}\left[\left(x^{3}\right)^{2}+1\right]\left|\psi_{R}(0,0)\right|^{2} d x \\
& \left.>\frac{1}{\mu} \int_{T^{\mu}}\left|\psi_{R}(0,0)\right|^{2} d x=\frac{1}{\mu}\left|\psi_{R}(0,0)\right|^{2} \text { [Volume of } T^{\mu}\right] \\
& =\frac{1}{6}\left|\psi_{R}(0,0)\right|^{2} .
\end{aligned}
$$

Combining this estimate with $(25)$ gives

$$
\left\|u_{I}\right\|_{T^{\mu}, 1}>\frac{1}{6} \sqrt{6} \ln (\ln R) \text {. }
$$

Also, for $x \in T^{\mu}$ we have $\left|x^{3}\right| \leq 1$ and

$$
\begin{aligned}
|u(x)|^{2}+|\nabla u(x)|^{2} & =\frac{1}{\mu}\left[\left|x^{3} \psi_{R}\left(x^{1}, x^{2}\right)\right|^{2}+\left|x^{3} \nabla \psi_{R}\left(x^{1}, x^{2}\right)\right|^{2}+\left|\psi_{R}\left(x^{1}, x^{2}\right)\right|^{2}\right] \\
& \leq \frac{2}{\mu}\left[\left|\psi_{R}\left(x^{1}, x^{2}\right)\right|^{2}+\left|\nabla \psi_{R}\left(x^{1}, x^{2}\right)\right|^{2}\right] .
\end{aligned}
$$

Let $\Delta$ denote the isosceles triangle that is the base of $T^{\mu}$ for $0<\mu<1$. Then with (26) we obtain

$$
\begin{aligned}
\|u\|_{T^{\mu}, 1}^{2} & \leq \frac{2}{\mu} \int_{T^{\mu}}\left[\left|\psi_{R}\left(x^{1}, x^{2}\right)\right|^{2}+\left|\nabla \psi_{R}\left(x^{1}, x^{2}\right)\right|^{2}\right] d x \\
& =\frac{2}{\mu} \int_{\Delta} \mu\left(1-x^{1}-x^{2}\right)\left[\left|\psi_{R}\left(x^{1}, x^{2}\right)\right|^{2}+\left|\nabla \psi_{R}\left(x^{1}, x^{2}\right)\right|^{2}\right] d x^{1} d x^{2} \\
& \leq 2 \int_{\Delta}\left[\left|\psi_{R}\left(x^{1}, x^{2}\right)\right|^{2}+\left|\nabla \psi_{R}\left(x^{1}, x^{2}\right)\right|^{2}\right] d x^{1} d x^{2} \\
& \leq 2\left\|\psi_{R}\right\|_{\Re^{2}, 1}^{2}<8 \pi,
\end{aligned}
$$

so that by $(30)$

$$
\left\|u-u_{I}\right\|_{T^{\mu}, 1} \geq\left\|u_{I}\right\|_{T^{\mu}, 1}-\|u\|_{T^{\mu}, 1}>\frac{1}{6} \sqrt{6} \ln (\ln R)-\sqrt{8 \pi}
$$

and $\left\|u-u_{I}\right\|_{T^{\mu}, 1} \rightarrow \infty$ uniformly for $0<\mu<1$ as $R \rightarrow \infty$.

On the other hand, with $D^{j}$ denoting $\frac{\partial}{\partial x^{j}}$ and $\tilde{x}=\left(x^{1}, x^{2}\right)$, we have

$$
\begin{aligned}
|u|_{T^{\mu}, 2}^{2}= & \int_{T^{\mu}}\left\{\left|D^{1} D^{1} u\right|^{2}+\left|D^{2} D^{2} u\right|^{2}+\left|D^{3} D^{3} u\right|^{2}\right. \\
& \left.\quad+2\left|D^{1} D^{2} u\right|^{2}+2\left|D^{1} D^{3} u\right|^{2}+2\left|D^{2} D^{3} u\right|^{2}\right\} d x \\
= & \frac{1}{\mu} \int_{T^{\mu}}\left\{\left|x^{3} D^{1} D^{1} \psi_{R}\right|^{2}+\left|x^{3} D^{2} D^{2} \psi_{R}\right|^{2}+2\left|x^{3} D^{1} D^{2} \psi_{R}\right|^{2}\right. \\
& \left.+2\left|D^{1} \psi_{R}\right|^{2}+2\left|D^{2} \psi_{R}\right|^{2}\right\} d x \\
= & \frac{1}{\mu} \int_{\Delta} \int_{0}^{\mu\left(1-x^{1}-x^{2}\right)}\left\{\left(x^{3}\right)^{2}\left[\psi_{R}(\tilde{x})\right]_{\tilde{x}, 2}^{2}+2\left|\nabla \psi_{r}(\tilde{x})\right|^{2}\right\} d x \\
= & \frac{1}{\mu} \int_{\Delta}\left[\frac{1}{3}\left(x^{3}\right)^{3}\left[\psi_{R}(\tilde{x})\right]_{\tilde{x}, 2}^{2}+2 x^{3}\left|\nabla \psi_{r}(\tilde{x})\right|^{2}\right]_{0}^{\mu\left(1-x^{1}-x^{2}\right)} d x^{1} d x^{2} \\
\leq & \frac{1}{\mu}\left\{\frac{1}{3} \mu^{3}\left|\psi_{R}\right|_{\Delta, 2}^{2}+2 \mu\left\|\psi_{R}\right\|_{\Delta, 1}^{2}\right\} \\
\leq & \frac{1}{3} \mu^{2}\left|\psi_{R}\right|_{\Re^{2}, 2}^{2}+2\left\|\psi_{R}\right\|_{\Re^{2}, 1}^{2} .
\end{aligned}
$$


With (26) and (27), this estimate yields

$$
|u|_{T^{\mu}, 2}<\sqrt{\frac{1}{3} \pi \mu^{2} R^{2}+8 \pi}
$$

and $|u|_{T^{\mu}, 2}<6$ for fixed $R$ and sufficiently small $\mu$.

Given $Y>0$, pick $R>e$ such that $\frac{1}{6} \sqrt{6} \ln (\ln R)-\sqrt{8 \pi}>6 Y$ and then pick $\mu$ with $0<\mu<1$ such that $\sqrt{\frac{1}{3} \pi \mu^{2} R^{2}+8 \pi}<6$. Then by (32) and (34),

$$
\left\|u-u_{I}\right\|_{T^{\mu}, 1}>6 Y>Y|u|_{T^{\mu}, 2} .
$$

Inequality (24) is satisfied, so there is no a priori estimate of the form (23) having the same constant $C$ for all tetrahedrons $T^{\mu}$ with $0<\mu<1$.

For the case of the rectangular boxes $Q^{\mu}$, we again define $u(x)$ by (28). Then for $0<\mu<1$,

$$
u_{I}(x)=\sum_{j=1}^{4} u_{I}^{(j)}(x)=\frac{1}{\sqrt{\mu}} \sum_{j=1}^{4} P_{j}(x) \psi_{R}\left(q_{j}\right),
$$

where $P_{1}(x)=\left(1-x^{1}\right)\left(1-x^{2}\right) x^{3}, \quad P_{2}(x)=x^{1}\left(1-x^{2}\right) x^{3}, \quad P_{3}=\left(1-x^{1}\right) x^{2} x^{3}$, $P_{4}(x)=x^{1} x^{2} x^{3}, q_{1}=(0,0), q_{2}=(1,0), q_{3}=(0,1)$, and $q_{4}=(1,1)$.

Short calculations yield for $j=1, \ldots, 4$,

$$
\begin{aligned}
\left\|u_{I}^{(j)}\right\|_{Q^{\mu}, 1}^{2} & =\frac{1}{\mu}\left|\psi_{R}\left(q_{j}\right)\right|^{2}\left\|P_{j}\right\|_{Q^{\mu}, 1}^{2} \\
& =\frac{1}{\mu}\left|\psi_{R}\left(q_{j}\right)\right|^{2}\left(\frac{7}{27} \mu^{3}+\frac{1}{9} \mu\right)=\left|\psi_{R}\left(q_{j}\right)\right|^{2}\left(\frac{7}{27} \mu^{2}+\frac{1}{9}\right) .
\end{aligned}
$$

Equations (35) and (25) imply

$$
\left\|u_{I}^{(1)}\right\|_{Q^{\mu}, 1}^{2}>\frac{1}{9}\left|\psi_{R}(0,0)\right|^{2}=\frac{1}{9}[\ln (\ln R)]^{2},
$$

while for $j=2,3,4$ and $0<\mu<1$,

$$
\left\|u_{I}^{(j)}\right\|_{Q^{\mu}, 1}=\left|\psi_{R}\left(q_{j}\right)\right| \sqrt{\frac{7}{27} \mu^{2}+\frac{1}{9}} \leq\left|\psi_{R}\left(q_{j}\right)\right| .
$$

By the definition of $\psi_{R}$,

$$
\psi_{R}\left(q_{2}\right)=\psi_{R}(1,0)=\frac{1}{2 \pi} \int_{e}^{R} \frac{1}{\rho \ln \rho} \int_{0}^{2 \pi} e^{i \rho \cos \theta} d \theta d \rho,
$$

while $\psi_{R}\left(q_{3}\right)$ and $\psi_{R}\left(q_{4}\right)$ are given by this formula with $\cos \theta$ replaced by $\sin \theta$ and $\cos \theta+\sin \theta=\sqrt{2} \sin (\theta+\pi / 4)$, respectively. We have

$$
\int_{0}^{2 \pi} e^{i \rho \cos \theta} d \theta=\int_{0}^{2 \pi} e^{i \rho \sin \theta} d \theta=2 \pi J_{0}(\rho),
$$

with $J_{0}$ the Bessel function of the first kind and order zero. (See any discussion of Bessel functions, such as M. Abramowitz and I. A. Stegun [9, pp. 360 and 364].) Moreover, there is a constant $k$ such that for $\rho \geq e$,

$$
\left|J_{0}(\rho)\right| \leq \frac{k}{\sqrt{\rho}} \text { and }\left|J_{0}(\sqrt{2} \rho)\right| \leq \frac{k}{\sqrt{\rho}},
$$


so that for $j=2,3,4$,

$$
\begin{aligned}
\left|\psi_{R}\left(q_{j}\right)\right| & \leq k \int_{e}^{R} \frac{1}{\rho^{3 / 2} \ln \rho} d \rho \\
& <k \int_{e}^{R} \rho^{-3 / 2} d \rho<\frac{2 k}{\sqrt{e}}<2 k .
\end{aligned}
$$

With (37) we obtain for $j=2,3,4$,

$$
\left\|u_{I}^{(j)}\right\|_{Q^{\mu}, 1}<2 k
$$

which, with (36), gives

$$
\begin{aligned}
\left\|u_{I}\right\|_{Q^{\mu}, 1} & \geq\left\|u_{I}^{(1)}\right\|_{Q^{\mu}, 1}-\sum_{j=2}^{4}\left\|u_{I}^{(j)}\right\|_{Q^{\mu}, 1} \\
& >\frac{1}{3} \ln (\ln R)-6 k .
\end{aligned}
$$

On the other hand, calculations (31) and (33) carried out for $Q^{\mu}$ in place of $T^{\mu}$ give with (26) and (27)

$$
\|u\|_{Q^{\mu}, 1} \leq \sqrt{2}\left\|\psi_{R}\right\|_{\Re^{2}, 1}<\sqrt{8 \pi}
$$

and

$$
|u|_{Q^{\mu}, 2} \leq \sqrt{\frac{1}{3} \mu^{2}\left|\psi_{R}\right|_{\Re^{2}, 2}^{2}+2\left\|\psi_{R}\right\|_{\Re^{2}, 1}^{2}} \leq \sqrt{\frac{1}{3} \pi \mu^{2} R^{2}+8 \pi} .
$$

Given $Y>0$, we pick $R>e$ such that $\frac{1}{3} \ln (\ln R)-6 k-\sqrt{8 \pi}>6 Y$ and then pick $\mu$ so that $\sqrt{\frac{1}{3} \pi \mu^{2} R^{2}+8 \pi}<6$. Then, $\left\|u-u_{I}\right\|_{Q^{\mu}, 1} \geq\left\|u_{I}\right\|_{Q^{\mu}, 1}-\|u\|_{Q^{\mu}, 1}>\frac{1}{3} \ln (\ln R)-6 k-\sqrt{8 \pi}>6 Y>Y|u|_{Q^{\mu}, 2}$, and, consequently, there is no constant $C$ such that

$$
\left\|u-u_{I}\right\|_{Q^{\mu}, 1} \leq C|u|_{Q^{\mu}, 2}
$$

for all $u \in C^{2}\left(Q^{\mu}\right)$ and $0<\mu<1$.

\section{ACKNOWLEDGMENT}

The author wishes to express his gratitude to Randy Bank for suggesting this topic.

\section{BIBLIOGRAPHY}

1. I. Babuška and A. K. Aziz, On the angle condition in the finite element method, SIAM J. Numer. Anal. 13 (1976), 214-226.

2. P. Jamet, Estimations d'erreur pour des éléments finis droits presque dégénerés, RAIRO Anal. Numér. 10 (1976), 43-60.

3. E. Barnhill and J. A. Gregory, Interpolation remainder theory from Taylor expansions on triangles, Numer. Math. 25 (1976), 401-408.

4. J. A. Gregory, Error bounds for linear interpolation on triangles, The Mathematics of Finite Elements and Applications, II (J. R. Whiteman, ed.), Academic Press, London, 1976, pp. 163-170.

5. M. H. Schultz, Spline analysis, Prentice-Hall, New York, 1973. 
6. L. Dechevski and E. Quak, On the Bramble-Hilbert lemma, Numer. Funct. Anal. Optim. 11 (1990), 485-495.

7. T. Dupont and R. Scott, Constructive polynomial approximation in Sobolev spaces, Recent Advances in Numerical Analysis (C. de Boor and G. H. Golub, eds.), Academic Press, New York, 1978, pp. 31-44.

8. N. A. Shenk, An elementary derivation of finite element error estimates, submitted to SIAM Rev.

9. M. Abramowitz and I. A. Stegun, eds., Handbook of mathematical functions, Dover, New York, 1964.

Department of Mathematics, University of California, San Diego, la Jolla, CaliforNIA 92093 